\title{
Total Energy Shaping of a Class of Underactuated Port-Hamiltonian Systems using a New Set of Closed-Loop Potential Shape Variables*
}

\author{
Christopher Renton ${ }^{1}$, Yik Ren $\mathrm{Teo}^{2}$ and Tristan Perez ${ }^{3}$
}

\begin{abstract}
This paper proposes a method for designing setpoint regulation controllers for a class of underactuated mechanical systems in Port-Hamiltonian System (PHS) form. A new set of potential shape variables in closed loop is proposed, which can replace the set of open loop shape variablesthe configuration variables that appear in the kinetic energy. With this choice, the closed-loop potential energy contains free functions of the new variables. By expressing the regulation objective in terms of these new potential shape variables, the desired equilibrium can be assigned and there is freedom to reshape the potential energy to achieve performance whilst maintaining the PHS form in closed loop. This complements contemporary results in the literature, which preserve the openloop shape variables. As a case study, we consider a robotic manipulator mounted on a flexible base and compensate for the motion of the base while positioning the end effector with respect to the ground reference. We compare the proposed control strategy with special cases that correspond to other energy shaping strategies previously proposed in the literature.
\end{abstract}

\section{INTRODUCTION}

A mechanical system with configuration variables $\mathbf{q} \in$ $\mathcal{Q}$ and conjugate momenta $\mathbf{p} \triangleq \partial \mathcal{L} / \partial \dot{\mathbf{q}} \in \mathcal{P}$, where $\mathcal{L}$ is the Lagrangian, is called fully actuated if the forces, $\tau \in \mathcal{F}$, produced by actuator configuration is such that $\operatorname{dim} \mathcal{F}=\operatorname{dim} \mathcal{P}$. If $\operatorname{dim} \mathcal{F}<\operatorname{dim} \mathcal{P}$ the system is said to be underactuated [1].

For fully-actuated mechanical systems, set-point regulation can be achieved by reshaping the potential energy such that it attains its minimum at the desired equilibrium. A positive definite potential energy function is chosen for the closed-loop system, and the control law can be found by matching the dynamics of the open-loop and desired closedloop systems [2].

If the mechanical system is modelled as a Port Hamiltonian System (PHS $)^{1}$, the more general method of Interconnection and Damping Assignment Passivity Based Control (IDA-PBC) allows not only potential energy shaping, but also kinetic energy shaping-that is, total energy shaping [4]. The matching conditions must satisfy a PDE in terms of both kinetic and potential energy, and the solution of

*This work has been supported by Boeing Research and Technology Australia (BR\&TA) through a collaborative research grant.

${ }^{1}$ C. Renton is with the School of Engineering, University of Newcastle, Australia christopher.renton at uon.edu.au

${ }^{2} \mathrm{Y}$. Teo is with the School of Engineering, University of Newcastle, Australia yikren.teo at uon.edu.au

${ }^{3} \mathrm{~T}$. Perez is with the School of Engineering, University of Newcastle, Australia Tristan.Perez at newcastle.edu.au and the Centre for Ships and Ocean Structures (CeSOS), Norwegian University of Science and Technology, Trondheim, Norway. [3].

${ }^{1}$ Also referred to as Port-Controlled Hamiltonian Systems in the literature this PDE provides the control law [4]. For underactuated mechanical systems, this PDE admits solutions only for a class of achievable total energy in closed loop. Such a PDE, in general, is difficult to solve.

As discussed in [5, p. 21], shape variables are configuration variables that appear in the open-loop kinetic energy. The remaining set of configuration variables are called $e x$ ternal variables. Underactuated mechanical systems can be classified according to which shape variables have actuation [5]. In [6] and [7], the control of a class of mechanical systems with unactuated shape variables ${ }^{2}$ is considered. The controllers are designed to preserve the open-loop shape variables in the closed-loop kinetic energy. A constructive method is proposed in [8] to solve the matching PDE for systems with underactuation degree one $(\operatorname{dim} \mathcal{F}=\operatorname{dim} \mathcal{P}-$ 1 ), and more recently in [9], this approach is extended to reduce the problem of solving the non-homogeneous kinetic energy PDE to a simpler problem of finding a transformation of the momentum state. Both approaches in [8], [9] also choose to preserve the shape variables in the closed loop.

In this paper, we consider a class of mechanical systems with unactuated external variables with stable dynamics. For this class of systems, potential energy shaping can be applied. This implements a partial state-feedback, that is, the external variables do not appear in the control law. The stability of the closed loop system relies on the passivity of the unactuated variables [2]. A performance issue arises if the dynamics in the unactuated variables are relatively slow, since there is no direct control authority in these channels. Hence, one can attempt to shape the total energy giving additional freedom to the designer. The performance of these controllers, however, may be limited if the open-loop shape variables are preserved in the closed loop.

Our modest contribution is, therefore, a method to design set-point regulation controllers for the above mentioned class of underactuated mechanical systems in PHS form. We do so by proposing a new set of potential shape variables in closed loop. Here, the meaning of the word potential is twofold. On the one hand, it means there is an option to use these variables as shape variables in the closed loop. On the other hand, even though these variables are related to the kinetic energy, they can be used to shape the potential energy. We show that the closed-loop potential energy contains free functions of these variables. By expressing the regulation objective in terms of the potential shape variables, we can

\footnotetext{
${ }^{2}$ With some abuse of terminology, we will refer to a configuration variable, $q_{j}$, as unactuated when no generalised force acts on the derivative of its conjugate momentum, namely $\dot{p}_{j}=d\left(\partial \mathcal{L} / \partial \dot{q}_{j}\right) / d t$.
} 
assign the desired equilibrium and have the freedom to reshape the potential energy to achieve performance whilst maintaining the PHS form in closed loop. As a case study, we consider a robotic manipulator mounted on a flexible base, and compensate for motion of the base while positioning the end effector with respect to the ground reference. We compare our control strategy with special cases that correspond to other energy shaping strategies previously proposed in the literature.

\section{A CLASS OF PORT HAMILTONIAN SYSTEMS}

We consider the following model of a simple mechanical system:

$$
\left[\begin{array}{c}
\dot{\mathbf{p}} \\
\dot{\mathbf{q}}
\end{array}\right]=\left\{\left[\begin{array}{cc}
\mathbf{0} & -\mathbf{I} \\
\mathbf{I} & \mathbf{0}
\end{array}\right]-\left[\begin{array}{cc}
\mathbf{D} & \mathbf{0} \\
\mathbf{0} & \mathbf{0}
\end{array}\right]\right\}\left[\begin{array}{c}
\frac{\partial \mathcal{H}(\mathbf{p}, \mathbf{q})}{\partial \mathbf{p}} \\
\frac{\partial \mathcal{H}(\mathbf{p}, \mathbf{q})}{\partial \mathbf{q}}
\end{array}\right]+\left[\begin{array}{c}
\mathbf{G} \\
\mathbf{0}
\end{array}\right] \boldsymbol{\tau} .
$$

The variable $\mathbf{q} \in \mathbb{R}^{n}$ gives the configuration of the system, and $\mathbf{p} \in \mathbb{R}^{n}$ is the conjugate momentum. We consider the control input $\tau \in \mathbb{R}^{m}$ (generalised forces). For typical underactuated systems, $m<n$.

We further assume that the configuration variables are partitioned as follows:

$$
\mathbf{q}=\left[\begin{array}{l}
\mathbf{q}_{e} \\
\mathbf{q}_{s}
\end{array}\right]
$$

where $\mathbf{q}_{s} \in \mathcal{Q}_{s}=\mathbb{R}^{m}$ is the vector of shape variables and $\mathbf{q}_{e} \in \mathcal{Q}_{e}=\mathbb{R}^{n-m}$ is the vector of external variables. The parameter $\mathbf{D}=\mathbf{D}^{\top} \in \mathbb{R}^{n \times n}$ is the positive definite damping, and $\mathbf{G} \in \mathbb{R}^{n \times m}$, with $\operatorname{rank}(\mathbf{G})=m$, is the input coupling matrix.

For the above class of systems, the shape variables are fully actuated, and we will assume,

$$
\mathbf{G}=\left[\begin{array}{c}
\mathbf{0}_{(n-m) \times m} \\
\mathbf{I}_{m \times m}
\end{array}\right] .
$$

If $\mathbf{G}$ is not in the form (3), then in some cases, it may be possible to transform the control variables to obtain the desired form.

The Hamiltonian function $\mathcal{H}(\mathbf{p}, \mathbf{q})$ is given by

$$
\mathcal{H}(\mathbf{p}, \mathbf{q})=\underbrace{\frac{1}{2} \mathbf{p}^{\top} \mathbf{M}^{-1}\left(\mathbf{q}_{s}\right) \mathbf{p}}_{\mathcal{T}\left(\mathbf{p}, \mathbf{q}_{s}\right)}+\mathcal{V}(\mathbf{q})
$$

where $\mathbf{M}\left(\mathbf{q}_{s}\right)=\mathbf{M}^{\top}\left(\mathbf{q}_{s}\right)>0$ is the mass matrix, which determines the open-loop kinetic energy $\mathcal{T}\left(\mathbf{p}, \mathbf{q}_{s}\right)$, and $\mathcal{V}(\mathbf{q})$ is the open-loop potential energy.

\section{SET-POINT REGULATION CONTROL FOR UNDERACTUATED MECHANICAL SYSTEMS}

Let us define $\mathbf{z}(\mathbf{q}) \in \mathcal{Z}$ as the potential shape variablesthe properties of this transformation are defined in Section III-D, and follow from the set of conditions for stability. We then consider set-point regulation of $\mathbf{z}$ to a desired equilibrium point $\mathbf{z}^{*}$.

\section{A. Closed-loop PHS}

Let the desired closed-loop PHS be

$$
\begin{array}{r}
{\left[\begin{array}{c}
\dot{\mathbf{p}} \\
\dot{\mathbf{q}}
\end{array}\right]=\left\{\left[\begin{array}{cc}
\mathbf{0} & -\mathbf{M}_{d}(\mathbf{z}) \mathbf{M}^{-1}\left(\mathbf{q}_{s}\right) \\
\mathbf{M}^{-1}\left(\mathbf{q}_{s}\right) \mathbf{M}_{d}(\mathbf{z}) & \mathbf{0}
\end{array}\right]\right.} \\
\left.-\left[\begin{array}{cc}
\mathbf{D}_{d} & \mathbf{0} \\
\mathbf{0} & \mathbf{0}
\end{array}\right]\right\}\left[\begin{array}{c}
\frac{\partial \mathcal{H}_{d}}{\partial \mathbf{p}_{d}} \\
\frac{\partial \mathcal{H}_{d}}{\partial \mathbf{q}}
\end{array}\right],
\end{array}
$$

where $\mathbf{D}_{d}=\mathbf{D}_{d}^{\top}>0$ is the desired damping, and the closedloop Hamiltonian is given by

$$
\mathcal{H}_{d}=\underbrace{\frac{1}{2} \mathbf{p}^{\top} \mathbf{M}_{d}^{-1}(\mathbf{z}) \mathbf{p}}_{\mathcal{T}_{d}(\mathbf{p}, \mathbf{z})}+\mathcal{V}_{d}(\mathbf{q}),
$$

where $\mathbf{M}_{d}(\mathbf{z})=\mathbf{M}_{d}^{\top}(\mathbf{z})>0$ is used to shape the kinetic energy $\mathcal{T}_{d}(\mathbf{p}, \mathbf{z})$. The potential energy $\mathcal{V}_{d}(\mathbf{q})$ is used to assign the desired closed-loop equilibrium, at which the potential energy attains its minimum.

\section{B. Matching}

To find a control law $\boldsymbol{\tau}$, we must match the $\dot{\mathbf{p}}$ and $\dot{\mathbf{q}}$ equations in (1) and (5). The $\dot{\mathbf{q}}$ equation is already matched by construction of (5) since

$$
\begin{aligned}
\dot{\mathbf{q}} & =\frac{\partial \mathcal{H}}{\partial \mathbf{p}}=\mathbf{M}^{-1}\left(\mathbf{q}_{s}\right) \mathbf{p} \\
& =\mathbf{M}^{-1}\left(\mathbf{q}_{s}\right) \mathbf{M}_{d}(\mathbf{z}) \mathbf{M}_{d}^{-1}(\mathbf{z}) \mathbf{p} \\
& =\mathbf{M}^{-1}\left(\mathbf{q}_{s}\right) \mathbf{M}_{d}(\mathbf{z}) \frac{\partial \mathcal{H}_{d}}{\partial \mathbf{p}} .
\end{aligned}
$$

The matching of $\dot{\mathbf{p}}$ yields

$$
\begin{aligned}
\dot{\mathbf{p}} & =-\mathbf{D M}^{-1}\left(\mathbf{q}_{s}\right) \mathbf{p}-\frac{\partial \mathcal{H}}{\partial \mathbf{q}}+\mathbf{G} \boldsymbol{\tau} \\
& =-\mathbf{D}_{d} \mathbf{M}_{d}^{-1}(\mathbf{z}) \mathbf{p}-\mathbf{M}_{d}(\mathbf{z}) \mathbf{M}^{-1}\left(\mathbf{q}_{s}\right) \frac{\partial \mathcal{H}_{d}}{\partial \mathbf{q}} .
\end{aligned}
$$

Since (8) consists of $n$ equations and $m<n$ unknown control forces, we need to satisfy the following additional $n-m$ constraints to find a solution for $\boldsymbol{\tau}$ :

$$
\begin{aligned}
\mathbf{G}^{\perp}\left\{\mathbf{D M}^{-1}\left(\mathbf{q}_{s}\right) \mathbf{p}-\mathbf{D}_{d} \mathbf{M}_{d}^{-1}(\mathbf{z}) \mathbf{p}\right. & \\
& \left.+\frac{\partial \mathcal{H}}{\partial \mathbf{q}}-\mathbf{M}_{d}(\mathbf{z}) \mathbf{M}^{-1}\left(\mathbf{q}_{s}\right) \frac{\partial \mathcal{H}_{d}}{\partial \mathbf{q}}\right\}=\mathbf{0},
\end{aligned}
$$

where $\mathbf{G}^{\perp}$ is any full-rank left annihilator of $\mathbf{G}$, that is, $\mathbf{G}^{\perp} \mathbf{G}=\mathbf{0}$ and $\operatorname{rank}\left(\mathbf{G}^{\perp}\right)=n-m$. If (9) is satisfied, the control law is given by

$$
\begin{aligned}
\boldsymbol{\tau}=\left(\mathbf{G}^{\mathbf{\top}} \mathbf{G}\right)^{-1} \mathbf{G}^{\boldsymbol{\top}} & \left\{\mathbf{D M}^{-1}\left(\mathbf{q}_{s}\right) \mathbf{p}-\mathbf{D}_{d} \mathbf{M}_{d}^{-1}(\mathbf{z}) \mathbf{p}\right. \\
+ & \left.\frac{\partial \mathcal{H}}{\partial \mathbf{q}}-\mathbf{M}_{d}(\mathbf{z}) \mathbf{M}^{-1}\left(\mathbf{q}_{s}\right) \frac{\partial \mathcal{H}_{d}}{\partial \mathbf{q}}\right\} .
\end{aligned}
$$

We can separate the matching equation, (9), into powers of $\mathbf{p}$, under the assumption that $\mathbf{D}$ and $\mathbf{D}_{d}$ are independent 
of p. Thus, a particular solution of (9) is obtained by solving the following equations:

$$
\begin{array}{r}
\mathbf{G}^{\perp}\left\{\frac{\partial \mathcal{V}}{\partial \mathbf{q}}-\mathbf{M}_{d}(\mathbf{z}) \mathbf{M}^{-1}\left(\mathbf{q}_{s}\right) \frac{\partial \mathcal{V}_{d}}{\partial \mathbf{q}}\right\}=\mathbf{0}, \\
\mathbf{G}^{\perp}\left\{\mathbf{D M}^{-1}\left(\mathbf{q}_{s}\right) \mathbf{p}-\mathbf{D}_{d} \mathbf{M}_{d}^{-1}(\mathbf{z}) \mathbf{p}\right\}=\mathbf{0}, \\
\mathbf{G}^{\perp}\left\{\frac{\partial \mathcal{T}}{\partial \mathbf{q}}-\mathbf{M}_{d}(\mathbf{z}) \mathbf{M}^{-1}\left(\mathbf{q}_{s}\right) \frac{\partial \mathcal{T}_{d}}{\partial \mathbf{q}}\right\}=\mathbf{0} .
\end{array}
$$

The objective is to choose $\mathbf{M}_{d}(\mathbf{z}), \mathcal{V}_{d}(\mathbf{q})$, and $\mathbf{D}_{d}$ to satisfy (11).

\section{Main result}

We consider choosing $\mathbf{z}$ to shape the total energy. Since the shape variables are actuated, $\mathbf{G}^{\perp} \frac{\partial \mathcal{T}}{\partial \mathbf{q}}=0$, then (11c) can be expressed as follows:

$$
\mathbf{G}^{\perp} \mathbf{M}_{d}(\mathbf{z}) \mathbf{M}^{-1}\left(\mathbf{q}_{s}\right) \frac{\partial^{\top} \mathbf{z}}{\partial \mathbf{q}} \frac{\partial \mathcal{T}_{d}}{\partial \mathbf{z}}=\mathbf{0} .
$$

This homogeneous PDE is solved for any $\mathbf{z}(\mathbf{q})=$ $\left[z_{1}(\mathbf{q}), \ldots, z_{m}(\mathbf{q})\right]^{\top}$ that satisfies

$$
\mathbf{G}^{\perp} \mathbf{M}_{d}(\mathbf{z}) \mathbf{M}^{-1}\left(\mathbf{q}_{s}\right) \frac{\partial z_{i}}{\partial \mathbf{q}}=\mathbf{0},
$$

$\forall i \in\{1, \ldots, m\}$. Thus, matching the kinetic energy has been reduced to finding $m$ functions of $\mathbf{q}$ from which we can form the shape variables for the closed-loop system. Note that (13) is simpler to solve than (12) since the latter involves the partial derivatives of $\mathbf{M}_{d}^{-1}(\mathbf{z})$. To solve (13), we propose a particular $\mathbf{z}$ and then try to solve an algebraic equation in $\mathbf{M}_{d}(\mathbf{z})$.

The potential energy PDE, (11a), can be separated into partial derivatives of external and shape variables,

$$
\begin{aligned}
& \mathbf{G}^{\perp}\left\{\frac{\partial \mathcal{V}}{\partial \mathbf{q}}-\mathbf{M}_{d}(\mathbf{z}) \mathbf{M}^{-1}\left(\mathbf{q}_{s}\right)\left[\begin{array}{c}
\frac{\partial \mathcal{V}_{d}}{\partial \mathbf{q}_{e}} \\
\mathbf{0}
\end{array}\right]\right. \\
&\left.-\mathbf{M}_{d}(\mathbf{z}) \mathbf{M}^{-1}\left(\mathbf{q}_{s}\right)\left[\begin{array}{c}
\mathbf{0} \\
\frac{\partial \mathcal{V}_{d}}{\partial \mathbf{q}_{s}}
\end{array}\right]\right\}=\mathbf{0}
\end{aligned}
$$

which is satisfied by $\mathcal{V}_{d}(\mathbf{q})=\mathcal{V}_{d e}(\mathbf{q})+\mathcal{V}_{d s}\left(\mathbf{q}_{s}\right)$ where $\mathcal{V}_{d e}(\mathbf{q})$ and $\mathcal{V}_{d s}\left(\mathbf{q}_{s}\right)$ are solutions of

$$
\begin{array}{r}
\mathbf{G}^{\perp}\left\{\frac{\partial \mathcal{V}}{\partial \mathbf{q}}-\mathbf{M}_{d}(\mathbf{z}) \mathbf{M}^{-1}\left(\mathbf{q}_{s}\right)\left[\begin{array}{c}
\frac{\partial \mathcal{V}_{d e}}{\partial \mathbf{q}_{e}} \\
\mathbf{0}
\end{array}\right]\right\}=\mathbf{0}, \\
\mathbf{G}^{\perp} \mathbf{M}_{d}(\mathbf{z}) \mathbf{M}^{-1}\left(\mathbf{q}_{s}\right)\left[\begin{array}{c}
\mathbf{0} \\
\frac{\partial \mathcal{V}_{d s}}{\partial \mathbf{q}_{s}}
\end{array}\right]=\mathbf{0 .}
\end{array}
$$

Equation (15a) can be simplified, by choosing

$$
\mathbf{G}^{\perp}=\left[\begin{array}{ll}
\mathbf{I}_{(n-m) \times(n-m)} & \mathbf{0}_{(n-m) \times m}
\end{array}\right],
$$

so that

$$
\frac{\partial \mathcal{V}_{d e}}{\partial \mathbf{q}_{e}}=\boldsymbol{\Gamma}^{-1}(\mathbf{q}) \frac{\partial \mathcal{V}}{\partial \mathbf{q}_{e}},
$$

where $\boldsymbol{\Gamma}(\mathbf{q})$ is the upper-left $(n-m) \times(n-m)$ block of $\mathbf{M}_{d}(\mathbf{z}) \mathbf{M}^{-1}\left(\mathbf{q}_{s}\right)$. The matrix $\boldsymbol{\Gamma}(\mathbf{q})$ is invertible since it is the $(n-m)^{\text {th }}$-order leading principal submatrix of a product of two square full rank matrices.
Equation (17) shows that the equilibrium points in the unactuated coordinates cannot be moved $^{3}$, as previously reported in [8] for systems with underactuation degree one, where $n-m=1, q_{e}$ and $\Gamma(\mathbf{q})$ are scalars and (17) may be integrated directly,

$$
\mathcal{V}_{d e}(\mathbf{q})=\int \frac{1}{\Gamma(\mathbf{q})} \frac{\partial \mathcal{V}}{\partial q_{e}} d q_{e}
$$

We can now design $\mathbf{M}_{d}(\mathbf{z})$, and thus $\boldsymbol{\Gamma}(\mathbf{q})$, so that $\mathcal{V}_{d e}(\mathbf{q})$ in (17) is positive definite in the external coordinates, $\mathbf{q}_{e}$, in a neighbourhood of its equilibrium, which we denote $\mathbf{q}_{e}^{*}$. This also suggests the possibility of extending this approach to problems with unstable open-loop dynamics in the unactuated coordinates. Since the product of two positive definite matrices, $\mathbf{M}_{d}(\mathbf{z}) \mathbf{M}^{-1}\left(\mathbf{q}_{s}\right)$, and thus $\boldsymbol{\Gamma}(\mathbf{q})$, is not necessarily positive definite (or even symmetric) it may be possible to design $\mathbf{M}_{d}(\mathbf{z})$ to produce a positive-definite potential $\mathcal{V}_{d e}(\mathbf{q})$ even for a non-positive-definite $\mathcal{V}(\mathbf{q})$. This is beyond the scope of the current paper.

Equation (15b) can be expressed in terms of $\mathbf{z}$ as follows:

$$
\mathbf{G}^{\perp} \mathbf{M}_{d}(\mathbf{z}) \mathbf{M}^{-1}\left(\mathbf{q}_{s}\right) \frac{\partial^{\top} \mathbf{z}}{\partial \mathbf{q}}\left[\begin{array}{c}
\mathbf{0} \\
\frac{\partial \mathcal{V}_{d z}}{\partial \mathbf{z}}
\end{array}\right]=\mathbf{0},
$$

which is satisfied for any free function $\mathcal{V}_{d z}(\mathbf{z})$, since $\mathbf{z}$ already satisfies (13). Therefore, a solution for the closedloop potential energy is given by

$$
\mathcal{V}_{d}(\mathbf{q})=\mathcal{V}_{d e}(\mathbf{q})+\mathcal{V}_{d z}(\mathbf{z}(\mathbf{q}))
$$

where $\mathcal{V}_{d e}(\mathbf{q})$ is a solution to $(15 \mathrm{a})$ and $\mathcal{V}_{d z}(\mathbf{z})$ is any free function of $\mathbf{z}$. We can then choose a function $\mathcal{V}_{d z}(\mathbf{z})$ so it is minimised at the desired equilibrium $\mathbf{z}^{*}$ and renders $\mathcal{V}_{d}(\mathbf{q})$ positive definite in a neighbourhood of $\mathbf{z}^{*}$.

Damping injection can be achieved by finding a matrix $\mathbf{D}_{d}$ which satisfies the algebraic constraints in (11b), that is,

$$
\mathbf{G}^{\perp} \mathbf{D}_{d} \mathbf{M}_{d}^{-1}(\mathbf{z})=\mathbf{G}^{\perp} \mathbf{D M}^{-1}\left(\mathbf{q}_{s}\right) .
$$

This determines the first $n-m$ rows (and due to symmetry the first $n-m$ columns) of $\mathbf{D}_{d}$.

\section{Stability}

Since the solution of $\mathcal{V}_{d e}(\mathbf{q})$ from (17) is minimised at $\mathbf{q}_{e}=\mathbf{q}_{e}^{*}$, and we choose $\mathcal{V}_{d z}(\mathbf{z})$ to be minimised at $\mathbf{z}=\mathbf{z}^{*}$, we can show that $\mathcal{V}_{d}(\mathbf{q})$ is minimised at $\mathbf{q}_{e}=\mathbf{q}_{e}^{*}$ and $\mathbf{z}=\mathbf{z}^{*}$.

Proposition 1: Given $\mathcal{V}_{d e}(\mathbf{q})>0, \forall \mathbf{q}_{e} \neq \mathbf{q}_{e}^{*}, \mathcal{V}_{d z}(\mathbf{z})>$ $0, \forall \mathbf{z} \neq \mathbf{z}^{*}$ and assuming that $\frac{\partial^{\top} \mathbf{z}}{\partial \mathbf{q}_{s}}$ is non-singular, then $\mathcal{V}_{d}(\mathbf{q})=\mathcal{V}_{d e}(\mathbf{q})+\mathcal{V}_{d z}(\mathbf{z})$ is minimised at $\mathbf{q}_{e}=\mathbf{q}_{e}^{*}$ and $\mathbf{z}=\mathbf{z}^{*}$.

Proof: The stationary points of $\mathcal{V}_{d}(\mathbf{q})$ are found by setting $\frac{\partial \mathcal{V}_{d}}{\partial \mathbf{q}}=\frac{\partial \mathcal{V}_{d e}}{\partial \mathbf{q}}+\frac{\partial^{\top} \mathbf{z}}{\partial \mathbf{q}} \frac{\partial \mathcal{V}_{d z}}{\partial \mathbf{z}}=\mathbf{0}$. Then, separating the

\footnotetext{
3 although the stability of the existing equilibrium points may be modified as shown in [6] and [7] for unstable systems.
} 
partial derivatives into $\mathbf{q}_{e}$ and $\mathbf{q}_{s}$ components, it follows from (17) that

$$
\begin{aligned}
\frac{\partial \mathcal{V}_{d}}{\partial \mathbf{q}_{e}} & =\boldsymbol{\Gamma}^{-1}(\mathbf{q}) \frac{\partial \mathcal{V}}{\partial \mathbf{q}_{e}}+\frac{\partial^{\top} \mathbf{z}}{\partial \mathbf{q}_{e}} \frac{\partial \mathcal{V}_{d z}}{\partial \mathbf{z}}=\mathbf{0}, \\
\frac{\partial \mathcal{V}_{d}}{\partial \mathbf{q}_{s}} & =\frac{\partial^{\top} \mathbf{z}}{\partial \mathbf{q}_{s}} \frac{\partial \mathcal{V}_{d z}}{\partial \mathbf{z}}=\mathbf{0} .
\end{aligned}
$$

Equation (23) is satisfied if and only if $\mathbf{z}=\mathbf{z}^{*}$ since $\frac{\partial^{\top} \mathbf{z}}{\partial \mathbf{q}_{o}}$ is full-rank. Equation (22) is satisfied for $\mathbf{q}_{e}=\mathbf{q}_{e}^{*}$ and $\mathbf{z}=\mathbf{z}^{*}$. To show there are no other stationary points, consider some $\mathbf{q}_{e} \neq \mathbf{q}_{e}^{*}$ and $\mathbf{z}=\mathbf{z}^{*}$ that satisfies (22). Then $\boldsymbol{\Gamma}^{-1}(\mathbf{q}) \frac{\partial \mathcal{V}}{\partial \mathbf{q}_{e}}=$ $\mathbf{0}$ for some $\mathbf{q}_{e} \neq \mathbf{q}_{e}^{*}$, but this is a contradiction since $\frac{\partial \mathcal{V}}{\partial \mathbf{q}_{e}}=\mathbf{0}$ if and only if $\mathbf{q}_{e}=\mathbf{q}_{e}^{*}$, and $\boldsymbol{\Gamma}(\mathbf{q})$ is full-rank. Therefore, $\mathcal{V}_{d}(\mathbf{q})$ is only stationary for $\mathbf{q}_{e}=\mathbf{q}_{e}^{*}$ and $\mathbf{z}=\mathbf{z}^{*}$.

To establish that $\mathcal{V}_{d}(\mathbf{q})$ is minimised, we note that it must be positive definite, since it is the sum of two positive definite functions.

The condition that $\frac{\partial^{\top} \mathbf{z}}{\partial \mathbf{q}_{\mathrm{s}}}$ is full rank requires $\mathbf{z}$ to be a function of all the elements of $\mathbf{q}_{s}$. This is not particularly restrictive, since if this were not the case, we could not assign any particular equilibrium point in all the $\mathbf{q}_{s}$ coordinates.

Note also that $\mathbf{z}$ may depend on elements of $\mathbf{q}_{e}$, but there is no difficulty in having the equilibrium specified by $\mathbf{q}_{e}=\mathbf{q}_{e}^{*}$ and $\mathbf{z}=\mathbf{z}^{*}$ which appears overdetermined. The reason for this, is that no choice of $\mathbf{z}$ which solves (13) can modify the location of the unactuated equilibrium points; so there is no conflict. We can use this feature to our advantage, as we show in the following section, since $\mathrm{z}$, being dependent on the unactuated coordinates, allows us to shape dynamics in $\mathbf{q}_{e}$.

We can now show that the closed-loop system is asymptotically stable.

Proposition 2: Consider the dynamics of the system (1) in closed-loop with the control law (10) and $\mathbf{M}_{d}(\mathbf{z}), \mathcal{V}_{d}(\mathbf{q})$ and $\mathbf{D}_{d}$ satisfy (11) and the conditions given in Proposition 1 hold. Then, the closed-loop system can be written as the PHS (5) which has an asymptotically stable equilibrium point at $\mathbf{p}=\mathbf{0}, \mathbf{q}_{e}=\mathbf{q}_{e}^{*}$ and $\mathbf{z}=\mathbf{z}^{*}$.

Proof: Using $\mathcal{H}_{d}$ as a Lyapunov candidate function, which is minimised at $\mathbf{p}=\mathbf{0}, \mathbf{q}_{e}=\mathbf{q}_{e}^{*}$ and $\mathbf{z}=\mathbf{z}^{*}$, we can compute its derivative with respect to time along the solutions of (5) and obtain

$$
\begin{aligned}
\dot{\mathcal{H}}_{d}(\mathbf{p}, \mathbf{q}) & =\frac{\partial^{\top} \mathcal{H}_{d}}{\partial \mathbf{p}} \dot{\mathbf{p}}+\frac{\partial^{\top} \mathcal{H}_{d}}{\partial \mathbf{q}} \dot{\mathbf{q}} \\
& =-\frac{\partial^{\top} \mathcal{H}_{d}}{\partial \mathbf{p}} \mathbf{D}_{d} \frac{\partial \mathcal{H}_{d}}{\partial \mathbf{p}} \leq 0
\end{aligned}
$$

which establishes stability. Asymptotic stability follows by applying the Invariance Principle. Since $\dot{\mathcal{H}}_{d}=0 \Longrightarrow \mathbf{p}=$ $\mathbf{0}$, then from (5) we have $\dot{\mathbf{p}}=-\mathbf{M}_{d}(\mathbf{z}) \mathbf{M}^{-1}\left(\mathbf{q}_{s}\right) \frac{\partial \mathcal{V}_{d}}{\partial \mathbf{q}}$ which is zero only for $\mathbf{q}_{e}=\mathbf{q}_{e}^{*}$ and $\mathbf{z}=\mathbf{z}^{*}$. Therefore the largest invariant set contained within $\left\{\mathbf{p}, \mathbf{q} \in \mathbb{R}^{n} \mid \dot{\mathcal{H}}_{d}=0\right\}$ is $\mathbf{p}=\mathbf{0}, \mathbf{q}_{e}=\mathbf{q}_{e}^{*}$ and $\mathbf{z}=\mathbf{z}^{*}$.

\section{CASE STUDY}

We consider the control of a robotic manipulator mounted on a flexible base. This is shown in Figure 1. The robot task consists of moving a heavy tool to a particular position in the workspace. The base has $1 \mathrm{DOF}, q_{1}$, and the robot arm has 2 DOFs, which correspond to rotation of the arm, $q_{2}$, and extension of the arm, $q_{3}$. Control torque $\tau_{m}$ acts to rotate the arm, and the control force $F_{A}$ acts to extend the arm.

Due to this configuration and the weight of the tool, the control system should be designed to compensate for motion of the base while positioning the end effector with respect to the ground reference.

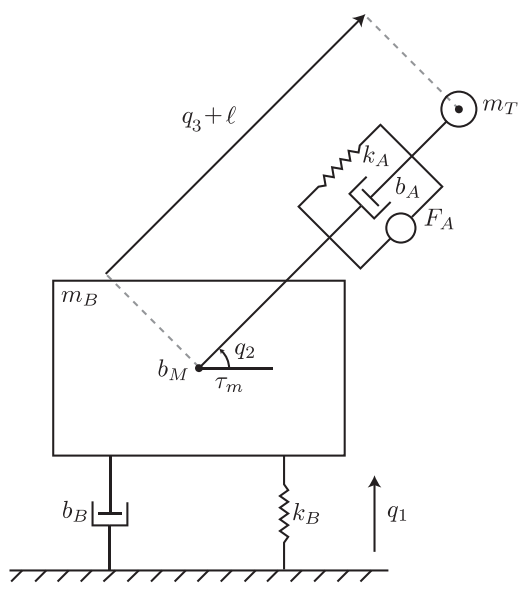

Fig. 1. Robotic manipulator mounted on a flexible base.

The open-loop mass matrix $\mathbf{M}\left(\mathbf{q}_{s}\right)$ is given by

$$
\begin{aligned}
& \mathbf{M}\left(\mathbf{q}_{s}\right)= \\
& {\left[\begin{array}{ccc}
m_{B}+m_{T} & m_{T}\left(q_{3}+\ell\right) \cos q_{2} & m_{T} \sin q_{2} \\
m_{T}\left(q_{3}+\ell\right) \cos q_{2} & m_{T}\left(q_{3}+\ell\right)^{2} & 0 \\
m_{T} \sin q_{2} & 0 & m_{T}
\end{array}\right] .}
\end{aligned}
$$

The open-loop potential energy $\mathcal{V}(\mathbf{q})$ is given by

$$
\begin{aligned}
\mathcal{V}(\mathbf{q})= & \frac{1}{2} k_{A} q_{3}^{2}+\frac{1}{2} k_{B} q_{1}^{2} \\
& +\left(m_{B}+m_{T}\right) g q_{1}+m_{T} g\left(q_{3}+\ell\right) \sin q_{2} .
\end{aligned}
$$

The input coupling matrix $\mathbf{G}$ and its left-annihilator $\mathbf{G}^{\perp}$ are given by

$$
\mathbf{G}=\left[\begin{array}{ll}
0 & 0 \\
1 & 0 \\
0 & 1
\end{array}\right], \quad \mathbf{G}^{\perp}=\left[\begin{array}{lll}
1 & 0 & 0
\end{array}\right] .
$$

Let the closed-loop mass matrix $\mathbf{M}_{d}(\mathbf{z})$ be parameterised as follows:

$$
\mathbf{M}_{d}(\mathbf{z})=\left[\begin{array}{lll}
a_{1} & a_{2} & a_{3} \\
a_{2} & a_{4} & a_{5} \\
a_{3} & a_{5} & a_{6}
\end{array}\right],
$$

and let the closed-loop damping $\mathbf{D}_{d}$ be parameterised as follows:

$$
\mathbf{D}_{d}=\left[\begin{array}{lll}
b_{1} & b_{2} & b_{3} \\
b_{2} & b_{4} & b_{5} \\
b_{3} & b_{5} & b_{6}
\end{array}\right]
$$


The regulation task is to position the tool, that is $\mathbf{x} \triangleq$ $[x, y]^{\top} \rightarrow \mathbf{x}^{*} \triangleq\left[x^{*}, y^{*}\right]^{\top}$ where,

$$
\begin{aligned}
& x=\left(q_{3}+\ell\right) \cos q_{2} \\
& y=q_{1}+\left(q_{3}+\ell\right) \sin q_{2}
\end{aligned}
$$

and $x^{*}$ and $y^{*}$ are the coordinates of the desired position in the workspace.

\section{A. Total energy shaping preserving shape variables}

We design a controller using total energy shaping, that preserves shape variables in the closed loop and recover the special case of potential energy shaping. This controller corresponds to a particular case of the controllers that can be designed with the method proposed in [4].

Substituting $\mathbf{z}=\mathbf{q}_{s}$ into (13) and assuming $\operatorname{det} \mathbf{M}(\mathbf{q})=$ $m_{B} m_{T}^{2}\left(q_{3}+\ell\right)^{2} \neq 0$, we have the constraints on the elements of $\mathbf{M}_{d}(\mathbf{z})$,

$$
\begin{aligned}
\left(a_{3} \sin q_{2}-a_{1}\right) m_{T}\left(q_{3}+\ell\right) \cos q_{2} & \\
+a_{2}\left(m_{B}+m_{T} \cos ^{2} q_{2}\right) & =0, \\
\left(a_{2} \cos q_{2}-a_{1}\left(q_{3}+\ell\right)\right) m_{T} \sin q_{2} & \\
+a_{3}\left(q_{3}+\ell\right)\left(m_{B}+m_{T} \sin ^{2} q_{2}\right) & =0 .
\end{aligned}
$$

By choosing $a_{1}>0$ as a design parameter, $a_{2}$ and $a_{3}$ can be expressed in terms of $a_{1}$,

$$
\begin{aligned}
a_{2} & =a_{1} \frac{m_{T}\left(q_{3}+\ell\right) \cos q_{2}}{m_{B}+m_{T}}, \\
a_{3} & =a_{1} \frac{m_{T} \sin q_{2}}{m_{B}+m_{T}}
\end{aligned}
$$

and $a_{4}, a_{5}$ and $a_{6}$ must be chosen to ensure $\mathbf{M}_{d}(\mathbf{z})>0$. We will choose $a_{5}=0$ to simplify the design. Then, the choice $a_{4}=\frac{a_{2}^{2}}{a_{1}}+\delta$ and $a_{6}=\frac{a_{3}^{2} a_{4}}{\delta}+\alpha$ leaves $\delta>0$ and $\alpha>0$ as design parameters which guarantees $\mathbf{M}_{d}(\mathbf{z})>0$.

From (18) and (20) we have

$$
\begin{array}{r}
\mathcal{V}_{d}(\mathbf{q})=\frac{m_{B}+m_{T}}{a_{1}}\left(\frac{1}{2} k_{B} q_{1}^{2}+\left(m_{B}+m_{T}\right) g q_{1}\right) \\
+\mathcal{V}_{d z}\left(q_{2}, q_{3}\right)
\end{array}
$$

where $\mathcal{V}_{d z}(\cdot, \cdot)$ is a free function. Since $\mathcal{V}_{d z}$ cannot depend on $q_{1}$, we choose

$$
\begin{aligned}
\mathcal{V}_{d z}\left(q_{2}, q_{3}\right)= & \frac{1}{2} k_{x}\left(\left(q_{3}+\ell\right) \cos q_{2}-x^{*}\right)^{2} \\
& +\frac{1}{2} k_{y}\left(\tilde{q}_{1}+\left(q_{3}+\ell\right) \sin q_{2}-y^{*}\right)^{2},
\end{aligned}
$$

where $\tilde{q}_{1} \triangleq-\frac{\left(m_{B}+m_{T}\right) g}{k_{B}}$ is the steady-state equilibrium position of $q_{1}$ and $k_{x}, k_{y}>0$ are free parameters. We have included $\tilde{q}_{1}$ to provide steady-state gravity compensation, in the absence of $q_{1}$.
Substituting (29) into (21) determines the first row and column of $\mathbf{D}_{d}$,

$$
\begin{aligned}
b_{1} & =\frac{b_{B}\left(-a_{2} \cos q_{2}+\left(q_{3}+\ell\right)\left(a_{1}-a_{3} \sin q_{2}\right)\right)}{m_{B}\left(q_{3}+\ell\right)}, \\
b_{2} & =\frac{b_{B}\left(a_{2}\left(q_{3}+\ell\right)-a_{4} \cos q_{2}\right)}{m_{B}\left(q_{3}+\ell\right)}, \\
b_{3} & =\frac{b_{B}\left(a_{3}-a_{6} \sin q_{2}\right)}{m_{B}} .
\end{aligned}
$$

Then, $b_{4}, b_{5}$ and $b_{6}$ must be chosen to satisfy $\mathbf{D}_{d}>0$. We will choose $b_{5}=0$ to simplify the design. Then, the choice $b_{4}=\frac{b_{2}^{2}}{b_{1}}+\gamma$ and $b_{6}=\frac{b_{3}^{2} b_{4}}{\gamma}+\beta$ leaves $\gamma>0$ and $\beta>0$ as design parameters which guarantees $\mathbf{D}_{d}>0$.

Note that if we set $a_{1}=m_{B}+m_{T}, a_{4}=m_{T}\left(q_{3}+\ell\right)^{2}$, $a_{5}=0$ and $a_{6}=m_{T}$ we have $\mathbf{M}_{d}(\mathbf{z})=\mathbf{M}(\mathbf{q})$ and we recover the special case of potential energy shaping.

To show stability, we note that $\mathcal{V}_{d e}(\mathbf{q})>0, \mathcal{V}_{d z}(\mathbf{z})>0$ and $\frac{\partial^{\top} \mathbf{z}}{\partial \mathbf{q}_{s}}=\mathbf{I}$, which satisfies the conditions in Proposition 1 . Since $\mathbf{M}(\mathbf{q})$ is singular at $q_{3}+\ell=0$, care should be taken. Assuming we have an initial condition $q_{3}(0)+\ell>0$ and target $q_{3}^{*}+\ell>0$, we can expect asymptotic stability by applying Proposition 2, since the trajectory of the states will not excite the singularity.

\section{B. Total energy shaping with potential shape variables}

We design a controller using total energy shaping by proposing potential shape variables which are compatible with the regulation task (30). Substituting $\mathbf{z}=\mathbf{x}$ into (13) and assuming $\operatorname{det} \mathbf{M}(\mathbf{q}) \neq 0$, we have the following constraints on the elements of $\mathbf{M}_{d}(\mathbf{z})$ :

$$
\begin{aligned}
& a_{3}\left(q_{3}+\ell\right) \cos q_{2}-a_{2} \sin q_{2}=0, \\
& a_{3}\left(q_{3}+\ell\right) \sin q_{2}-a_{2} \cos q_{2}=0 .
\end{aligned}
$$

The solution to (36) is given by $a_{2}=a_{3}=0$. Then, $a_{1}$, $a_{4}, a_{5}$ and $a_{6}$ are free parameters subject to $a_{1}, a_{4}>0$ and $a_{4} a_{6}>a_{5}^{2}$ which guarantees $\mathbf{M}_{d}(\mathbf{z})>0$.

From (18) and (20) we have

$$
\begin{aligned}
\mathcal{V}_{d}(\mathbf{q})=\frac{m_{B}+m_{T}}{a_{1}}\left(\frac{1}{2} k_{B} q_{1}^{2}+\left(m_{B}\right.\right. & \left.\left.+m_{T}\right) g q_{1}\right) \\
& +\mathcal{V}_{d z}(x, y),
\end{aligned}
$$

where $\mathcal{V}_{d z}(\cdot, \cdot)$ is a free function. Now we can choose

$$
\mathcal{V}_{d z}(x, y)=\frac{1}{2} k_{x}\left(x-x^{*}\right)^{2}+\frac{1}{2} k_{y}\left(y-y^{*}\right)^{2}
$$

where $k_{x}, k_{y}>0$ are free parameters.

Substituting (29) into (21) determines the first row and column of $\mathbf{D}_{d}$,

$$
\begin{aligned}
& b_{1}=\frac{a_{1} b_{B}}{m_{B}}, \\
& b_{2}=\frac{-b_{B}\left(a_{4} \cos q_{2}+a_{5}\left(q_{3}+\ell\right) \sin q_{2}\right)}{m_{B}\left(q_{3}+\ell\right)}, \\
& b_{3}=\frac{-b_{B}\left(a_{5} \cos q_{2}+a_{6}\left(q_{3}+\ell\right) \sin q_{2}\right)}{m_{B}\left(q_{3}+\ell\right)} .
\end{aligned}
$$


Then, $b_{4}, b_{5}$ and $b_{6}$ must be chosen to satisfy $\mathbf{D}_{d}>0$. We will choose $b_{5}=0$ to simplify the design. Then, the choice $b_{4}=\frac{b_{2}^{2}}{b_{1}}+\gamma$ and $b_{6}=\frac{b_{3}^{2} b_{4}}{\gamma}+\beta$ leaves $\gamma>0$ and $\beta>0$ as design parameters which guarantees $\mathbf{D}_{d}>0$.

Note that for this choice of $\mathbf{z}, \frac{\partial^{\top} \mathbf{z}}{\partial \mathbf{q}_{s}}$ is only full rank for $q_{3}+\ell \neq 0$, however this also corresponds to the singularity in $\mathbf{M}\left(\mathbf{q}_{s}\right)$ so we do not expect any reduction in the region of attraction compared to the previous case. We should note however, that while the equilibrium is unique in $\mathbf{z}$ coordinates, there are two solutions in $\mathbf{q}_{s}$ coordinates. These correspond to the cases of the extended arm $q_{3}+\ell>0$, and the inverted arm $q_{3}+\ell<0$, and the corresponding angles, $q_{2}$, which differ by $\pi$. Unlike the design in the previous section, where the equilibrium in $\mathbf{q}_{s}$ is specified, assuming that $q_{3}(0)+\ell>0$ and target $q_{3}^{*}+\ell>0$ is not sufficient to prevent the state trajectory from crossing the singularity. This may be overcome by constraining $q_{3}+\ell>0$, however, this is beyond the scope of the current paper.

\section{Simulation Results}

The plant parameters corresponding to the manipulator shown in Figure 1 are given in the Appendix. The flexibility of the base is exaggerated to appreciate the effect of the controllers.

Three control designs are considered:

i) Potential energy shaping, that is, $\mathbf{M}_{d}(\mathbf{z})=\mathbf{M}\left(\mathbf{q}_{s}\right)$ and $\mathbf{z}=\mathbf{q}_{s}$

ii) Total energy shaping preserving open-loop shape variables, that is $\mathbf{z}=\mathbf{q}_{s}$

iii) Total energy shaping with potential shape variables $\mathbf{z}=$ $\mathrm{x}$.

The controller parameters given in Table I. The initial condition was $\mathbf{p}(0)=\mathbf{q}(0)=\mathbf{0}$, and the target was located at $x=4 \mathrm{~m}$ and $y=3 \mathrm{~m}$.

Figures 2 and 3 show the simulation results. We can see that for the designs (i) and (ii), the $q_{2}$ and $q_{3}$ states converge quickly to their steady-state values, and the control action remains small thereafter. The natural base oscillation, however, causes large errors in the tool position, which the controller is not informed about. While additional freedom is available in the design (ii), it was still not possible to improve the transient response significantly over that achieved by only shaping potential energy.

The controller for case (iii) can be seen to be using the $q_{2}$ and $q_{3}$ states to actively compensate for motion in the base. The result is the tool error quickly converges to zero, while the controller continues to produce control forces which actively cancel the base motion at the tool position, until the natural motion of the base decays due to the dissipative forces in the base.

\section{CONCLUSIONS}

We have shown that by reducing the kinetic energy matching PDE (over the unknown elements of $\mathbf{M}_{d}$ ) to one involving potential shape variables $\mathbf{z}$, we have both simplified the design procedure for total energy shaping, and made it easier to design admissible closed-loop potential energy
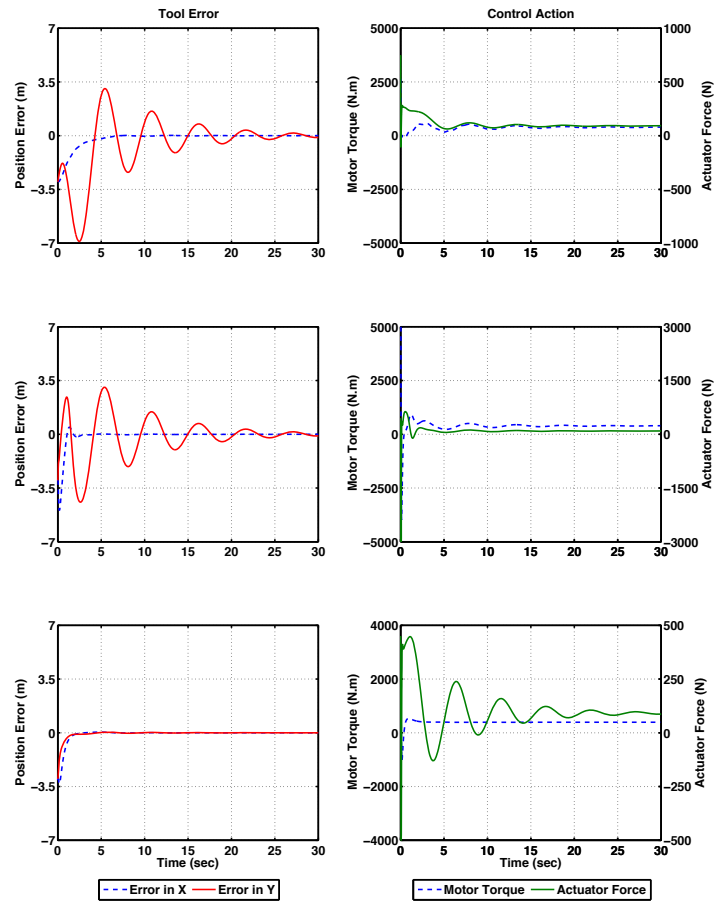

Fig. 2. Tool positioning error (left) and control action (right) for potential energy shaping (top), total energy shaping with $\mathbf{z}=\mathbf{q}_{s}$ (middle) and $\mathbf{z}=\mathbf{x}$ (bottom).
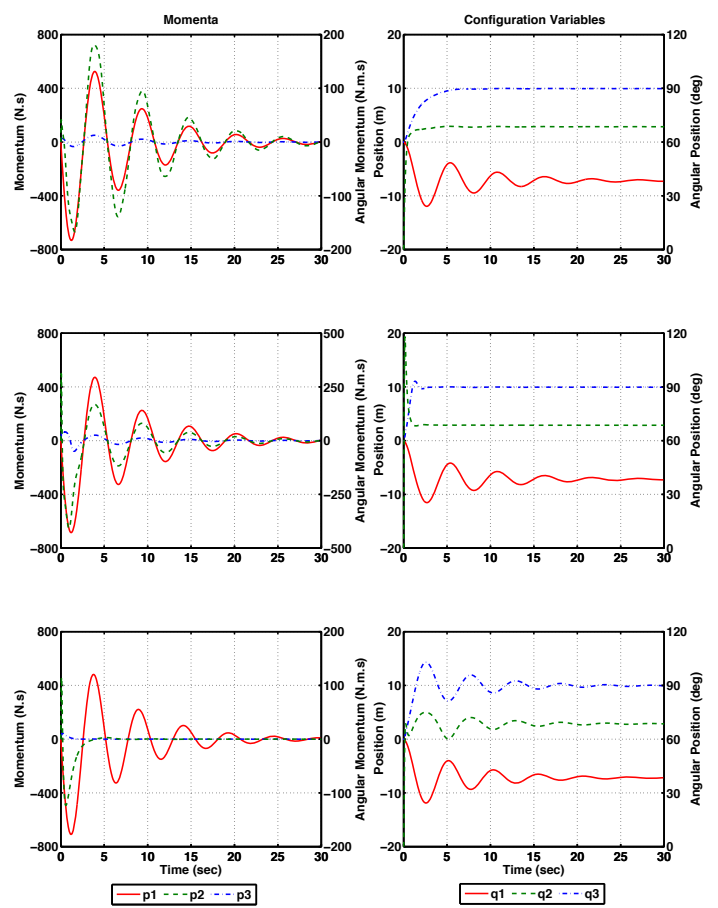

Fig. 3. State variables: momenta (left) and displacements (right) for potential energy shaping (top), total energy shaping with $\mathbf{z}=\mathbf{q}_{s}$ (middle) and $\mathbf{z}=\mathbf{x}$ (bottom). 
functions for this class of underactuated mechanical systems. Once $\mathbf{z}$ has been chosen, we are able to choose the various free functions of $\mathbf{z}$ in the elements of $\mathbf{M}_{d}(\mathbf{z})$, subject to algebraic constraints, rather than having to directly solve the kinetic energy PDE (11c).

In a case study, we have shown how this controller can be used for compensating vibration of a robotic manipulator mounted on a flexible unactuated base. The proposed controller shows improvement in performance relative to controllers based on potential energy shaping and total energy shaping preserving shape variables.

\section{APPENDIX}

The plant parameters are given by $m_{B}=100 \mathrm{~kg}, m_{T}=$ $10 \mathrm{~kg}, \ell=1 \mathrm{~m}, k_{A}=0 \mathrm{~N} / \mathrm{m}, k_{B}=150 \mathrm{~N} / \mathrm{m}$ and the openloop damping matrix is given by

$$
\mathbf{D}=\left[\begin{array}{ccc}
b_{B} & 0 & 0 \\
0 & b_{M} & 0 \\
0 & 0 & b_{A}
\end{array}\right]
$$

where $b_{B}=30 \mathrm{Ns} / \mathrm{m}, b_{M}=1 \mathrm{Nms} / \mathrm{rad}$ and $b_{A}=50 \mathrm{Ns} / \mathrm{m}$.

The controller parameters are given in Table I.

TABLE I

CONTROLLER PARAMETERS.

\begin{tabular}{cccc}
\hline Parameter & PE & TE $\left(\mathbf{z}=\mathbf{q}_{s}\right)$ & TE $(\mathbf{z}=\mathbf{x})$ \\
\hline \hline$a_{1}$ & $m_{B}+m_{T}$ & 50 & 50 \\
$a_{4}$ & $m_{T}\left(q_{3}+\ell\right)^{2}$ & $*$ & 5 \\
$a_{6}$ & $m_{T}$ & $*$ & 1 \\
$\alpha$ & N/A & 5 & N/A \\
$\delta$ & N/A & 1 & N/A \\
$b_{1}$ & 30 & $*$ & $*$ \\
$b_{4}$ & 450 & $*$ & $*$ \\
$b_{5}$ & 200 & 0 & 0 \\
$\gamma$ & N/A & 8 & 8 \\
$\beta$ & 250 & 10 & 10 \\
$k_{x}$ & 250 & 1500 & 1500 \\
$k_{y}$ & 250 & 1500 & 1500 \\
\hline
\end{tabular}

\section{REFERENCES}

[1] F. Bullo and A. D. Lewis, Geometric control of mechanical systems. Springer, 2005, vol. 49.

[2] R. Ortega, A. Loría, P. J. Nicklasson, and H. J. Sira-Ramírez, Passivitybased Control of Euler-Lagrange Systems: Mechanical, Electrical, and Electromechanical Applications. Springer Verlag, 1998.

[3] A. van der Schaft, L2-gain and passivity techniques in nonlinear control. London: Springer-Verlag, 2000.

[4] R. Ortega, A. Van Der Schaft, B. Maschke, and G. Escobar, "Interconnection and damping assignment passivity-based control of portcontrolled hamiltonian systems," Automatica, vol. 38, no. 4, pp. 585596, 2002.

[5] R. Olfati-Saber, "Nonlinear control of underactuated mechanical systems with application to robotics and aerospace vehicles," Ph.D. dissertation, Massachusetts Institute of Technology, 2000.

[6] F. Gomez-Estern, R. Ortega, F. Rubio, and J. Aracil, "Stabilization of a class of underactuated mechanical systems via total energy shaping," in Decision and Control, 2001. Proceedings of the 40th IEEE Conference on, vol. 2. IEEE, 2001, pp. 1137-1143.

[7] R. Ortega, M. Spong, F. Gómez-Estern, and G. Blankenstein, "Stabilization of a class of underactuated mechanical systems via interconnection and damping assignment," Automatic Control, IEEE Transactions on, vol. 47, no. 8, pp. 1218-1233, 2002.
[8] J. Acosta, R. Ortega, A. Astolfi, and A. Mahindrakar, "Interconnection and damping assignment passivity-based control of mechanical systems with underactuation degree one," Automatic Control, IEEE Transactions on, vol. 50, no. 12, pp. 1936-1955, 2005.

[9] G. Viola, R. Ortega, R. Banavar, J. Acosta, and A. Astolfi, "Total energy shaping control of mechanical systems: simplifying the matching equations via coordinate changes," Automatic Control, IEEE Transactions on, vol. 52, no. 6, pp. 1093-1099, 2007. 